\title{
Surface modification of as-synthesized lamellar mesostructured silica obtained by liquid crystal templating
}

\author{
C. Henrist, ${ }^{* a b}$ C. Vogels, ${ }^{c}$ A. Rulmont ${ }^{a}$ and R. Cloots ${ }^{a b}$ \\ ${ }^{a}$ SUPRATECS, University of Liège, B6 Sart Tilman, 4000 Liege, Belgium. \\ E-mail: catherine.henrist@ulg.ac.be; Fax: +324366 34 13; Tel: +32 43663438 \\ ${ }^{\boldsymbol{b}}$ CAT $\mu$, University of Liège, B6 Sart Tilman, 4000 Liège \\ ${ }^{c}$ SOLVAY S.A., rue de Ransbeek 310, 1120 Brussels, Belgium
}

Received (in Montpellier, France) 4th January 2005, Accepted 31st May 2005

First published as an Advance Article on the web 1st July 2005

We report a study of lamellar silica phase silylation, starting from as-synthesized silica, without the usual heat treatment step. Characterizations of the modified silica include X-ray diffraction, thermal analysis, electron microscopy and solid state NMR. Special attention is given to the possibility of keeping the lamellar organisation along with the elimination of the organic template.

\section{Introduction}

Surface modification of oxide substrates by grafting of siliconcontaining molecules is common, for micro-, meso- or nonporous materials. ${ }^{1-4}$ Silane grafting on the surface of lamellar mesostructured silica is of special interest since this modification makes the surface organophilic and enhances its compatibility with organic solvents or polymers for dispersion or catalytic purposes, as well as its stability with respect to atmospheric humidity. 5,6

The usual silylating procedures and characterization techniques of silylated porous substrates are not suitable for lamellar mesostructured silica. Indeed, for most three-dimensional porous materials, including the well-known MCM-41 mesoporous molecular sieve, the silylating step is done after a high temperature treatment, in order to burn out the organic template, if present, and above all activate the silanol groups located on the surface of the pores. ${ }^{7-10}$ Nevertheless, a lamellar mesostructure would be destroyed during this calcination step. ${ }^{11}$ Therefore, it is important to develop an alternative silylating method that is able to preserve the lamellar organisation.

The idea of modifying uncalcined mesoporous materials is not new, even for tridimensional porous substrates whose structure is preserved upon thermal treatment. An alternative post-treatment procedure was studied in order to achieve, in a single step, several goals: preparation of functionalized mesoporous materials with hydrophobic surfaces, removal of surfactant molecules from the mesopores, avoiding high temperature calcination or solvent extraction steps and avoiding structure shrinkage during the calcination process. Moreover, this technique showed a higher surface coverage of grafted ligands, compared to the silylation of calcined materials, especially in the case of hindered alkylsilanes. The first attempts were carried out by refluxing the uncalcined materials for $36 \mathrm{~h}$ in pure chlorosilane agent, then adding a small amount of pyridine for an additional $18 \mathrm{~h}$ reflux. ${ }^{12}$ Later, a dry and non-polar solvent, which is able to extract the template out of the interlayer space and free the surface silanol groups, has been proposed to drive the silylating reaction with a silylating agent, directly on as-synthesized mesostructured solids. ${ }^{13}$

Characterization of modified microporous oxides is usually done by gas adsorption measurements with molecules of wellknown size (toluene, $n$-hexane, water). Such experiments can establish whether modification occurs inside the pores or on the external surface and provide information about pore blocking, uniformity of surface coverage. ${ }^{14,15}$ However in the case of lamellar phases, this technique can be not suitable since the accessible surface is not delimited by walls organised into pores with a specific size and geometry. Other characterization techniques are thus envisaged in order to get information on the preservation of the lamellar organization and the grafting level of the silylating agent onto the substrate. X-Ray diffraction, thermal analysis, electron microscopy and solid state NMR constitute useful tools for the understanding and description of the modified lamellar silica structure.

We present here the results of an adapted silylating procedure applied to uncalcined lamellar mesostructured silica, synthesized in acidic or basic medium, and structural characterization of the modified solid phase obtained.

\section{Experimental}

\section{Synthesis of the lamellar mesostructured silica}

Dimethyldioctadecylammonium chloride $\left(2 \times \mathrm{C}_{18} \mathrm{Cl}, \mathrm{KaO}\right.$ Corp.) is dispersed in hot water $\left(50{ }^{\circ} \mathrm{C}\right)$ at a concentration of $3 \mathrm{wt} \%$ with axial stirring, until a homogeneous medium is obtained. In order to facilitate the dispersion, the surfactant is first melted on a hot plate then transferred into the pre-heated water under vigorous stirring and the resulting white-blueish suspension is aged for $30 \mathrm{~min}$ to complete the mesophase formation. Concentrated $\mathrm{HCl}$ or tetramethylammonium hydroxide (TMAOH, Merck) is then added dropwise to the mixture to adjust the $\mathrm{pH}$ to the desired value: 0 for acidic synthesis and 12 for basic synthesis. This results in an increase of the suspension turbidity. The silica precursor is then added to the template under stirring in a molar ratio surfactant $: \mathrm{Si}=$ 1 : 0.22 . Two silica precursors were used: sodium silicate solution with $25.5-28.5 \mathrm{wt} \% \mathrm{SiO}_{2}$ (Silicate $\mathrm{Na}$, Merck) and tetraethyl orthosilicate (TEOS, Hüls). Two combination are chosen: TEOS $/ 2 \times \mathrm{C}_{18} /$ acid and Silicate $\mathrm{Na} / 2 \times \mathrm{C}_{18} /$ basic.

The reacting mixture is kept under stirring at $50{ }^{\circ} \mathrm{C}$ for $21 \mathrm{~h}$ then filtered off, and washed several times with room temperature de-ionized water, until neutral. The recovered solid is dried in air at moderate temperature $\left(<50{ }^{\circ} \mathrm{C}\right)$ and ground for analysis. 
The lamellar mesostructured silica obtained under basic conditions (hereafter named "basic substrate") is made of negative silica layers intercalated with the alkylammonium template ions. The template is anchored by ionic bonding in the interlayer space.

The lamellar mesostructured silica obtained under acidic conditions (hereafter named "acidic substrate") is made of neutral silica layers intercalated with the alkylammonium chloride template molecules. The template is anchored as ions pairs by hydrogen-type bonding to the polar surface of the silica layers.

\section{Surface modification}

A direct template displacement method was developed to graft silane ligands on to the surface of uncalcined mesoporous silica prepared under acidic conditions. ${ }^{16,17}$ The organic surfactant can easily be recovered and re-used as the template of the mesoporous silicas. The high density of surface silanol groups in acid-synthesized silica leads to high loading of silanes. The mild reaction conditions and short reaction time lead to the preservation of the morphology.

Vartuli et al. ${ }^{18}$ have used hexamethyldisiloxane solvent or HMDS $\left(\left(\mathrm{CH}_{3}\right)_{3} \mathrm{Si}-\mathrm{O}-\mathrm{Si}\left(\mathrm{CH}_{3}\right)_{3}\right)$, in combination with the modifying agent trimethylsilyl chloride or TMSCl $\left(\left(\mathrm{CH}_{3}\right)_{3} \mathrm{Si}-\mathrm{Cl}\right)$. The procedure consists in dispersing $0.5 \mathrm{~g}$ of the as-synthesized mesostructured lamellar silica (hereafter named the substrate) in $15 \mathrm{~g}$ of HMDS mixed with $10 \mathrm{~g}$ of TMSCl. The reacting medium was refluxed overnight $(18-20 \mathrm{~h})$ then cooled down to room temperature and filtered on a Büchner funnel. The resulting powder is washed several times with acetone to eliminate solvent traces, then dried at moderate temperature in air.

\section{Structural characterization}

X-Ray diffraction patterns were collected in a Siemens D5000 powder diffractometer using Ni-filtered $\mathrm{Cu} K \alpha$ radiation. Thermal analysis data were recorded on a Netzsch STA 449C analyzer under air, with a heating ramp of $10{ }^{\circ} \mathrm{C} \mathrm{min}^{-1}$ and a gas flux of $50 \mathrm{ml} \mathrm{m^{-1 }}$. Transmission electron micrographs were obtained on a Philips CM100 microscope working at an acceleration voltage of $100 \mathrm{kV}$. The powders were simply deposited on a copper grid covered with a formvar thin film. The ${ }^{29} \mathrm{Si}$ MAS-NMR data were recorded in a Bruker Advance DSX400 (9.4T) spectrometer operating at $79.5 \mathrm{MHz}$. Quantitative spectra were obtained by performing one-pulse experiments with a $90^{\circ}$ pulse width of $5.5 \mu \mathrm{s}$. Quantitativity was checked by varying the delay time and an optimal value was found at $480 \mathrm{~s}$.

\section{Results and discussion}

The as-synthesized substrates have a different lamellar periodicity, which is visible in their X-ray diffraction pattern (fine curve on Fig. 1 and 2): the lamellar silica obtained in acidic conditions shows a $37.6 \AA$ periodicity while its basic counterpart exhibits a peak at $41.5 \AA$. This is attributed to a higher content of water, hydroxyl groups and/or organic species in the basic substrate, which was confirmed by thermal analysis and solid state NMR (not presented here). The synthesis in acidic medium from TEOS precursor leads to a solid phase characterized by a higher degree of silica network polycondensation, along with a more compressed organic layer located between the silica sheets. Acidic substrates are thus richer in silica, poorer in silanol groups and have a lower $d_{001}$ periodicity. Elemental detection by Energy Dispersive X-ray spectrometry, coupled to a scanning electron microscope, confirmed the lack of sodium and chloride ions in the basic substrates and therefore corroborates the interaction model dealing with a direct

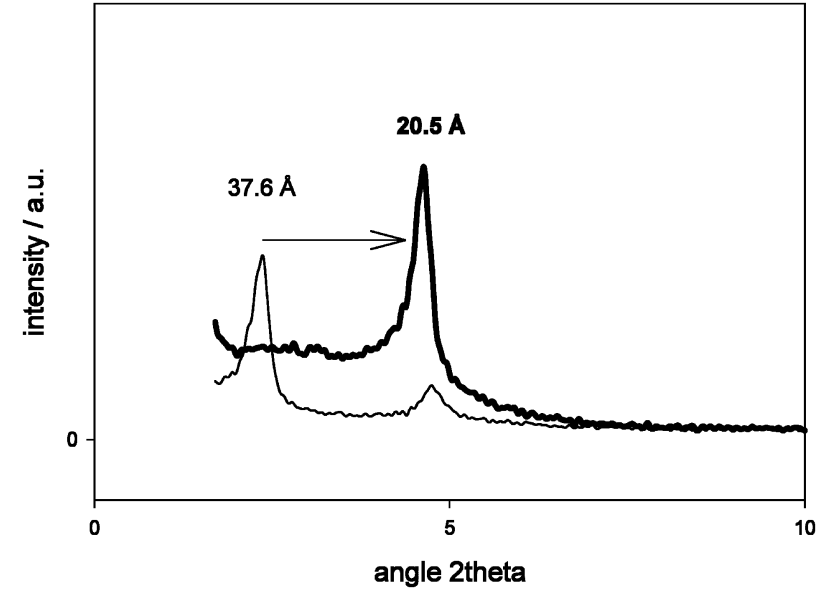

Fig. 1 X-Ray diffraction patterns of a silica obtained in the TEOS $2 \times \mathrm{C}_{18} \mathrm{Cl} /$ acid system. Fine curve: as-synthesized substrate. Bold curve substrate modified by TMSCl in HMDS.

interaction between the cationic surfactant and the negatively charged silica network $\left(\mathrm{S}^{+} \mathrm{I}^{-}\right)$. Counter-ions from $2 \times \mathrm{C}_{18} \mathrm{Cl}$ and sodium silicate are washed out after synthesis as $\mathrm{NaCl}$. On the other hand, chloride is detected in acidic substrates, which confirms the interaction model $\mathrm{S}^{+} \mathrm{X}^{-} \mathrm{I}^{0}$, where $\mathrm{S}^{+}$stands for the cationic surfactant, $\mathrm{X}^{-}$for the chloride ion and $\mathrm{S}^{0}$ for the neutral silica network.

The two types of substrates were successfully modified and characterized by X-ray diffraction, thermal analysis, transmission electron microscopy and MAS-NMR.

The periodicity of lamellar stacking is changed after the silylating step (bold curve on Fig. 1 and 2): the modified acidic substrate shows now a larger lamellar periodicity than the modified basic substrate. The reducing of the lamellar periodicity is due to the replacement of the large, intercalated template molecules by smaller trimethylsilyl groups, grafted on the surface silanol groups. It is important to note that the lamellar organisation is preserved in both cases.

Thermal analysis measurements show that this difference in periodicity is not ascribable to a larger amount of fixed agent that would lead to a thicker deposited layer. A precise analysis of thermal analysis curves provides several pieces of information related to the internal structure of the modified substrates (Fig. 3 and 4). First, the total elimination of the intercalated template is visualized since it caused intense and complex exothermal peaks between $150{ }^{\circ} \mathrm{C}$ and $500{ }^{\circ} \mathrm{C}$ in the

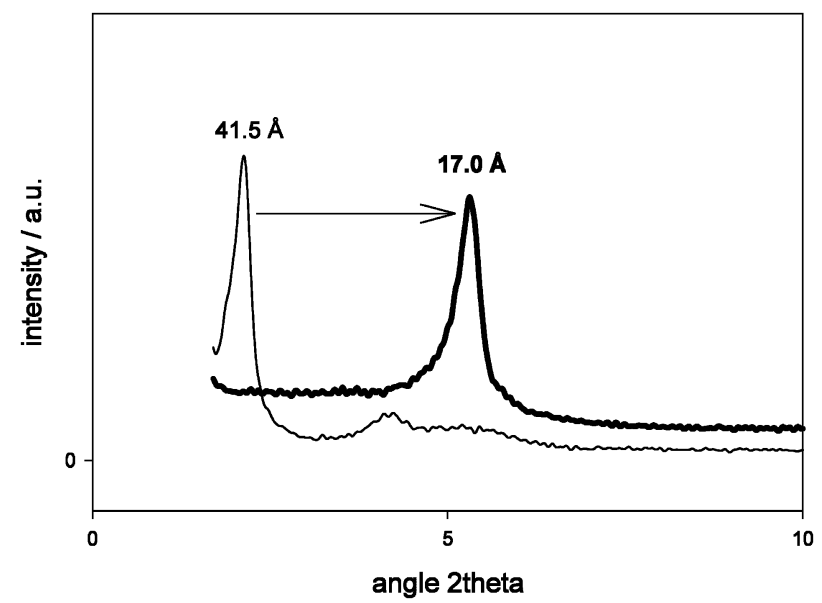

Fig. 2 X-Ray diffraction patterns of a silica obtained in the TEOS $2 \times \mathrm{C}_{18} \mathrm{Cl} /$ base system. Fine curve: as-synthesized substrate. Bold curve: substrate modified by TMSCl in HMDS. 


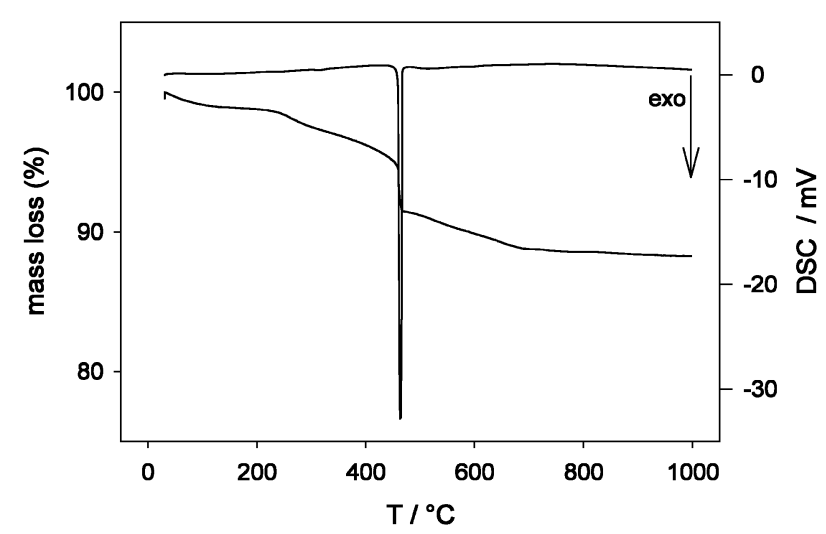

Fig. 3 TGA and DSC curves of an acidic modified substrate. Mass loss associated with the exothermal peak: $3.5 \mathrm{wt} \%$.

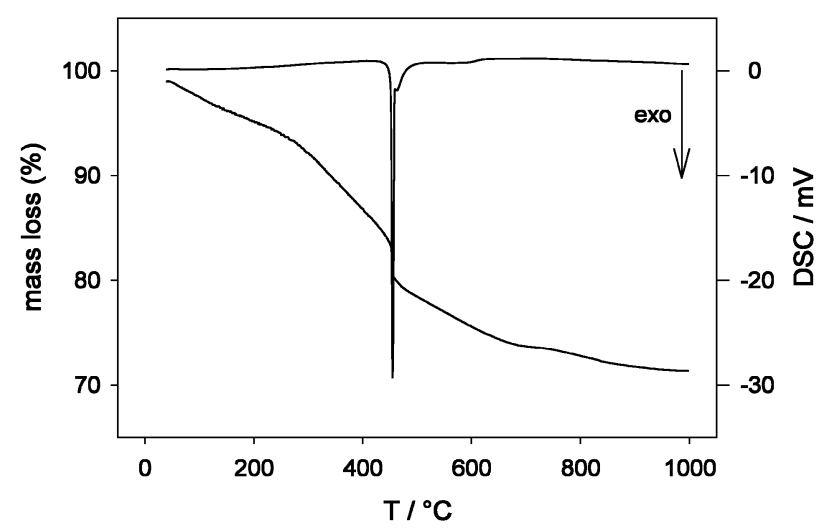

Fig. 4 TGA and DSC curves of a basic modified substrate. Mass loss associated with the exothermal peak: $4.0 \mathrm{wt} \%$.

as-synthesized substrate ${ }^{19}$ (data not shown here), along with a mass loss of 40 to $60 \mathrm{wt} \%$. Second, the narrow exothermal peak related to a very small mass loss confirms the grafting of the modifying agent. The amount of fixed agent is quite similar in both cases: it is evaluated as $3.5 \mathrm{wt} \%$ for the acidic substrate and $4.0 \mathrm{wt} \%$ for the basic substrate. The grafted trimethylsilyl group burns at very high temperature $\left(\sim 450{ }^{\circ} \mathrm{C}\right)$, which indicates a covalent link rather than simple physisorption on the surface. ${ }^{20,21}$ Lastly, this combustion reaction corresponds to the oxidation of trimethylsilyl into $\mathrm{SiO}_{2}$ with evolution of $\mathrm{CO}_{2}$ and $\mathrm{H}_{2} \mathrm{O}$. This oxidative elimination is achieved in a single step within a narrow temperature range. No endothermic dehydroxylation process is observed, although the sample undergoes a progressive mass loss along the entire heating ramp. The substrate obtained under acidic conditions leads to a silica-richer solid phase after silylating, since the mass residue at $1000{ }^{\circ} \mathrm{C}$ is higher in this case. This difference is not attributed to a higher TMS content, therefore it must arise from a different level of hydration and condensation of the silica network (confirmed by Si NMR measurements, not shown here) as well as thicker silica layers. Fig. 5 proposes a summary scheme of the lamellar packing periodicity, before and after silylating.

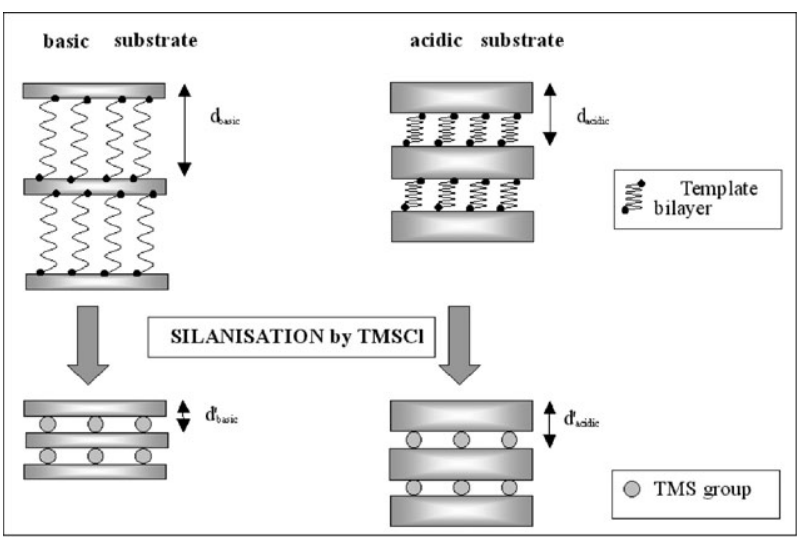

Fig. 5 Summary scheme showing the lamellar packing periodicity $\left(d_{001}\right)$ of acidic and basic substrates, before and after silylating by TMSCl. Before silylating, it is supposed that the basic substrates are characterized by thinner silica layers and more elongated intercalated template molecules.

The amount of modifying agent fixed by an oxide is usually presented in mmol per gram of substrate. Here we consider that the TMS groups are converted into a molecular layer of $\mathrm{SiO}_{2}$ during heat treatment: therefore the mass gain by atmospheric oxygen fixation has to be taken into account. When submitted to heat, each TMS group undergoes a $3 / 2 \mathrm{O}$ gain and a $3 \mathrm{CH}_{3}$ loss. The mass balance is $+(3 / 2 \times 16)-(3 \times 15)=$ $-21 \mathrm{~g}$ per burned TMS mole. One gram of silylated substrate loses $4 \mathrm{wt} \%$, which equals $0.04 \mathrm{~g}$. The corresponding quantity of TMS is thus $0.04 / 21=0.0019 \mathrm{~mol}$. Regarding the molecular weight of TMS $\left(73 \mathrm{~g} \mathrm{~mol}^{-1}\right)$, the amount of fixed agent can be calculated as $2.21 \mathrm{mmol} \mathrm{g}^{-1}$ of pure substrate. Table 1 summarizes the main properties of the two silylated substrates.

Solid state NMR is a powerful technique to investigate the grafting of silylating agents, to determine the amount as well as the type of bonding and reactive sites. ${ }^{22,23} \mathrm{~A}$ sample of substrate obtained in the TEOS $/ 2 \times \mathrm{C}_{18} /$ acid was characterized by Si MAS-NMR, before and after silylating. ${ }^{24}$ Fig. 6 shows the repartition of the different $\mathrm{Si}$ atom types in this sample. Before surface modification, the intercalated lamellar silica is mainly constituted of $\mathrm{Q}_{3}$ and $\mathrm{Q}_{4}$ silicon atoms in equivalent quantity. The initial silica network can be considered as thin lamellae with $50 \%$ of "inner $\mathrm{Si}$ " atoms $\left(\mathrm{Q}_{4}\right)$ and $50 \%$ of "surface $\mathrm{Si}$ " $\left(\mathrm{Q}_{3}\right)$ atoms bearing an $\mathrm{OH}$ group. This reveals the very thinness of the layers, which have a very high surface : volume ratio. After the grafting of TMS, an intense peak appears in the spectrum, corresponding to the TMS signal. This new peak counts for $25 \%$ of the total spectrum area, while the $\mathrm{Q}_{3}$ peak decreases to $9.5 \%$ and the $\mathrm{Q}_{4}$ increases to $65.3 \%$. In the modified substrate, there are thus 6.8 times more $\mathrm{Q}_{4}$ atoms than $\mathrm{Q}_{3}$. It can be calculated that $3 / 4$ of the surface $\mathrm{SiOH}$ groups have fixed a TMS molecule.

A virtual numbering of the different $\mathrm{Si}$ atom types is presented in Table 2, based on an ideal silica layer containing 1000 inner Si and 1000 surface Si. All the missing $\mathrm{Q}_{3}$ atoms are converted into $\mathrm{Q}_{4}$ atoms by fixation of a TMS group, and not by condensation with water evolving for example.

We can make an estimation of the specific surface of the substrate available for the silylating. The surface area occupied

Table 1 Comparison of the structural characteristics of two lamellar silica's obtained in acidic and basic conditions. Behavior under silylating treatment by TMSCl

\begin{tabular}{|c|c|c|c|c|c|}
\hline $\begin{array}{l}\text { Silica } \\
\text { precursor }\end{array}$ & $\begin{array}{l}\text { Synthesis pH of the } \\
\text { substrate (before silylating) }\end{array}$ & $\begin{array}{l}d_{\max } \text { of as-synthesized } \\
\text { substrate } / \AA\end{array}$ & $\begin{array}{l}d_{\max } \text { of substrate } \\
\text { modified by } \mathrm{TMSCl} / \AA\end{array}$ & $\begin{array}{l}\text { TMS fixed } \\
\text { amount (wt } \%)\end{array}$ & $\begin{array}{l}\mathrm{SiO}_{2} \text { content (dry residue } \\
\left.\text { at } 1000{ }^{\circ} \mathrm{C}\right)(\mathrm{wt} \%)\end{array}$ \\
\hline TEOS & 0 & 37.6 & 20.5 & 3.5 & 88.3 \\
\hline $\mathrm{Na}$ Silicate & 12 & 41.5 & 17.0 & 4.0 & 71.4 \\
\hline
\end{tabular}




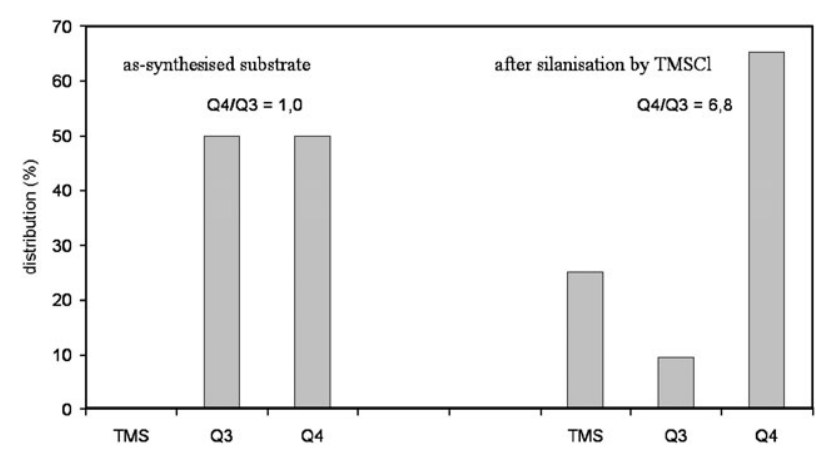

Fig. 6 Distribution of silicon atoms in lamellar mesostructured silica obtained under acidic conditions, before and after modifying by TMSCl.

by a TMS group is around $s=41 \AA^{2} .^{25}$ One gram of substrate can fix $2.21 \mathrm{mmol}$ of TMS, which corresponds to a total surface of:

$$
\begin{aligned}
S_{\text {tot }}= & n(\text { TMS fixed per gram }) \times N_{\mathrm{A}} \times s=2.21 \times 10^{-3} \\
& \times 6.024 \times 10^{23} \times 41 \times 10^{-20}=545.8 \mathrm{~m}^{2} \mathrm{~g}^{-1} .
\end{aligned}
$$

This value is very high especially for a large molecule such as $\mathrm{TMSCl}$ that cannot enter very narrow pores. Such a huge specific area indicates not only a very good dispersion level of the lamellae in the HMDS solvent, and their extremely high aspect ratio, but also a total elimination of the template, which would hinder the access to the surface reaction sites.

Despite the fact that substrates obtained in basic conditions are richer in silanol surface groups, they show the same grafting capacity as the acidic substrates. The spatial hindering of the grafted TMS groups seems to be the limiting factor of the surface modification reaction.

Transmission electron microscopy provides additional evidence of the preservation of the lamellar organisation during the silylating process. Fig. 7 presents four revealing micrographs of the layered morphology of modified substrates. Micrographs (a) and (c) show large, nearly single layers of one micron in width. Note the very poor mechanical rigidity due to the extreme thinness of the silica lamellae, indicated by the visible folding. Micrographs (b) and (d) are taken at higher magnification, in zones where the stacking of several layers can be observed, especially on the border of the aggregates. The conservation of this regular stacking gives rise to the detected peak in the X-ray diffraction pattern.

It is easy to understand that these lamellae have very poor cohesion with each other, since the interlayer space is filled with the trimethylsilyl groups, pointing towards the next layer, and interacting only by the way of van der Waals forces. This lamellar structure should be easily dispersed in a non-polar medium, such as polyolefins. In contrast, they should remain stacked together in a polar environment in order to minimize the contact with their organophilic surfaces.

Table 2 Virtual numbering of the distribution of different $\mathrm{Si}$ atom types in a lamellar mesostructured silica, before and after treatment by TMSCl (acidic substrate)

\begin{tabular}{lll}
\hline & Before silylating & $\begin{array}{l}\text { After } \\
\text { silylating }\end{array}$ \\
\hline $\mathrm{Q}_{4} / \mathrm{Q}_{3}$ & 1 & 6.8 \\
$N\left(\mathrm{Q}_{3}\right)$ & $1000 \rightarrow 50 \%$ & 257 \\
$N\left(\mathrm{Q}_{4}\right)$ & $1000 \rightarrow 50 \%$ & 1743 \\
$N\left(\mathrm{Q}_{3}\right.$ converted & & $743 \rightarrow 74.3 \%$ of \\
into $\left.\mathrm{Q}_{4}\right)$ & & surface silanols \\
$N$ (fixed TMS) & & 743 \\
$N$ total & 2000 & 2743 \\
$\%$ (TMS) calculated & & $(743 / 2743) \times 100=27 \%$ \\
$\%$ (TMS) observed & & $25 \%$ \\
\hline
\end{tabular}
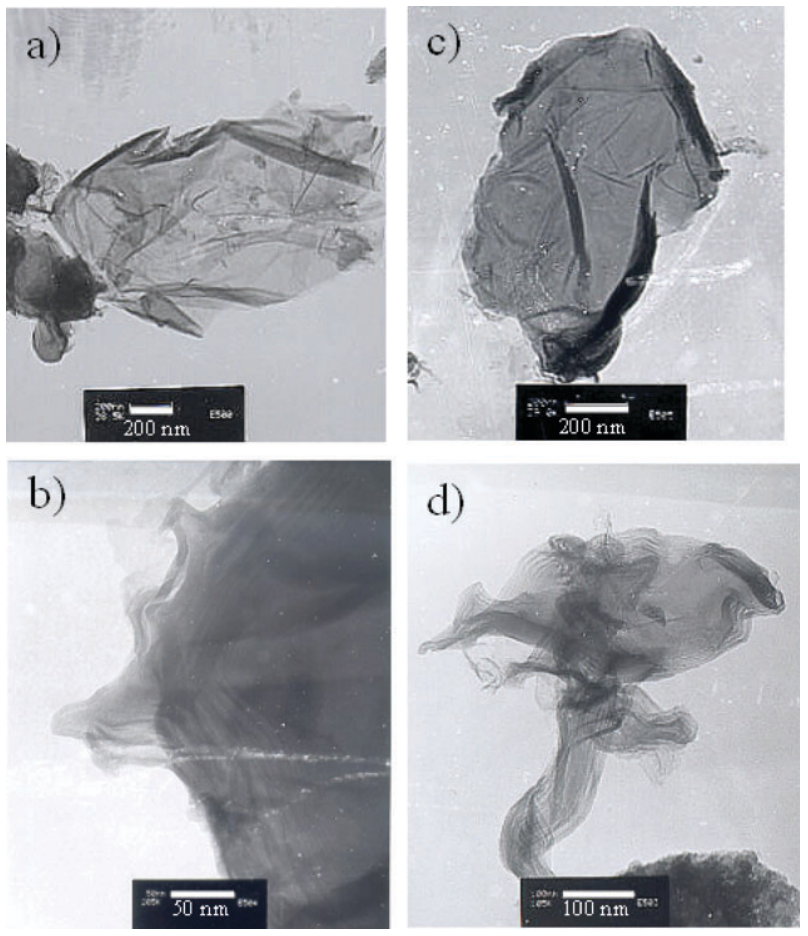

Fig. 7 TEM micrographs TEM of lamellar silica modified by TMSCl in HMDS. Pictures (a) and (b) acidic substrate. Pictures (c) and (d) basic substrate.

\section{Conclusions}

The modification of as-synthesized lamellar mesostructured silicas is efficient in a solvent able to extract the intercalated template out of the interlayer space, at the same time as the grafting reaction. We have succeeded in modifying both acidic and basic silica substrates, although they exhibit different structural characteristics. The chloride ion released by the $\mathrm{TMSCl}$ modifying agent is used during the extraction of the template out of the basic lamellar silica: the alkylammonium, initially anchored by ionic binding to the silica surface, captures the chloride counter-ion to form a neutral ion pair that can be extracted by the HMDS.

All the characterization techniques show that the lamellar structure is preserved. The quantitative analysis of the trimethylsilyl fixation has revealed that three quarters of the surface silanols had fixed a TMS group and that the dissociation of the individual lamellae in the solvent during the reaction has optimised the contact area between the modifying agent and the silica to be treated. The reaction is therefore very complete, and is only limited by spatial hindering considerations of the TMS groups.

\section{Acknowledgements}

Solid state NMR spectra were collected by Prof. Jean Grandjean at the NMR centre of the University of Liège (CREMANUlg). The authors thank Prof. Grandjean for useful discussion of the results.

\section{References}

1 N. R. E. N. Impens, P. van der Voort and E. Vansant, Microporous Mesoporous Mater., 1999, 28, 217.

2 P. Cool and E. F. Vansant, Trends Phys. Chem., 1999, 7, 145-158.

3 R. Singh and P. K. Dutta, Microporous Mesoporous Mater., 1999, 32, 29-35.

4 H. Yang, G. Zhang, X. Hong and Y. Zhu, Microporous Mesoporous Mater., 2004, 68(1-3), 119-125.

5 D. Brunel, Microporous Mesoporous Mater., 1999, 27, 329-344. 
6 A. Corma, V. Fornés, J. M. Guil, S. Pergher, Th. L. M. Maesen and J. G. Buglass, Microporous Mesoporous Mater., 2000, 38, 301-309.

7 C. P. Jaroniec, M. Kruk, M. Jaroniec and A. Sayari, J. Phys. Chem. B, 1998, 102, 5503.

8 X. S. Zhao and G. Q. Lu, J. Phys. Chem. B, 1998, 102, 1556.

9 J. S. Beck, J. C. Vartuli, W. J. Roth, M. E. Leonowicz, C. T. Kresge, K. D. Schmitt, C. T. Chu, D. H. Olson, E. W. Sheppard, S. B. McCullen, J. B. Higgins and J. L. Schlenker, J. Am. Chem. Soc., 1992, 114, 10834.

10 X. S. Zhao, G. Q. Lu and X. Hu, Microporous Mesoporous Mater., 2000, 41, 37-47.

11 A. W. Xu, Y. P Cai, L. Z. Zhang and J. C. Yu, Adv. Mater., 2002, 14(15), 1064-1068

12 V. Antochshuk and M. Jaroniec, Chem. Commun., 1999, 2373-2374.

13 P. Sutra, F. Fajula, D. Brunel, P. Lentz, G. Daelen and J. B. Nagy, Colloid Surf. A, 1999, 158, 21-27.

14 V. Antochshuk, M. Kruk and M. Jaroniec, J. Phys. Chem. B, 2003, 107(43), 11900-11906.

15 X. S. Zhao, G. Q. Lu, X. Hu, Adsorption Science and Technology, Proceedings of the Pacific Basin Conference on Adsorption Science and Technology, 2nd, Brisbane, Australia, May 14-18, 2000 (2000).
16 H. P. Lin, Y. H. Liu, C. P. Kao, S. B. Liu and C. Y. Mou, Zeolites and Mesoporous Materials at the Dawn of the 21st Century, Stud. Surf. Sci. Catal., 2001, 135, 4733-4739.

17 H. P. Lin, L. Y. Yang, C. Y. Mou, S. B. Liu and H. K. Lee, New J. Chem., 2000, 24(5), 253-255.

18 J. C. Vartuli, K. D. Schmitt, C. T. Kresge, W. J. Roth, M. E. Leonowicz, S. B. McCullen, S. D. Hellring, J. S. Beck, J. L. Schlenker, D. H. Olson and E. W. Sheppard, Chem. Mater., 1994, 6, 2317.

19 C. Henrist, I. Garcia Cano, M. A. Rodriguez, A. H. de Aza, R. Cloots, A. Rulmont and C. Vogels, Compos. Sci. Technol., 2003, 63, 1127-1131

20 A. J. Jeong, S. M. Goo and D. P. Kim, J. Sol-Gel Sci. Technol., 2000, 19(1/2/3), 483-487.

21 D. W. Hua and D. M. Smith, Langmuir, 1992, 8(11), 2753-7.

22 B. Lindlar, M. Luechinger, A. Roethlisberger, M. Haouas, G. Pirngruber, A. Kogelbauer and R. Prins, J. Mater. Chem., 2002, 12(3), 528-533.

23 B. J. S. Johnson and A. Stein, Inorg. Chem., 2001, 40(4), 801-808.

24 X. S. Zhao, G. Q. Lu, A. K. Whittaker, G. J. Millar and H. Y. Zhu, J. Phys. Chem. B, 1997, 101, 6525-6531.

25 B. H. Wouters, T. Chen, M. Dewilde and P. J. Grobet, Microporous Mesoporous Mater., 2001, 44-45, 453. 